\title{
A study of inter-lab and inter-platform agreement of DNA microarray data Huixia Wang ${ }^{1}$, Xuming He${ }^{1}$, Mark Band ${ }^{2}$, Carole Wilson ${ }^{2}$ and Lei Liu*2
}

Address: ${ }^{1}$ Department of Statistics, University of Illinois at Urbana-Champaign, 101 Illini Hall, 725 South Wright Street, Champaign, Illinois 61820, USA and ${ }^{2}$ W. M. Keck Center for Comparative and Functional Genomics, University of Illinois at Urbana-Champaign, 1201 W. Gregory Drive, Urbana, Illinois 61801, USA

Email: Huixia Wang - hwang22@uiuc.edu; Xuming He - x-he@uiuc.edu; Mark Band - markband@uiuc.edu; Carole Wilson - cawilson@uiuc.edu; Lei Liu* - leiliu@uiuc.edu

* Corresponding author

Published: II May 2005

BMC Genomics 2005, 6:7| doi:10.1|86/|47|-2|64-6-7|
Received: 30 July 2004

Accepted: II May 2005

This article is available from: http://www.biomedcentral.com/I47I-2164/6/7I

(C) 2005 Wang et al; licensee BioMed Central Ltd.

This is an Open Access article distributed under the terms of the Creative Commons Attribution License (http://creativecommons.org/licenses/by/2.0), which permits unrestricted use, distribution, and reproduction in any medium, provided the original work is properly cited.

\begin{abstract}
As gene expression profile data from DNA microarrays accumulate rapidly, there is a natural need to compare data across labs and platforms. Comparisons of microarray data can be quite challenging due to data complexity and variability. Different labs may adopt different technology platforms. One may ask about the degree of agreement we can expect from different labs and different platforms. To address this question, we conducted a study of inter-lab and inter-platform agreement of microarray data across three platforms and three labs. The statistical measures of consistency and agreement used in this paper are the Pearson correlation, intraclass correlation, kappa coefficients, and a measure of intra-transcript correlation. The three platforms used in the present paper were Affymetrix GeneChip, custom cDNA arrays, and custom oligo arrays. Using the within-platform variability as a benchmark, we found that these technology platforms exhibited an acceptable level of agreement, but the agreement between two technologies within the same lab was greater than that between two labs using the same technology. The consistency of replicates in each experiment varies from lab to lab. When there is high consistency among replicates, different technologies show good agreement within and across labs using the same RNA samples. On the other hand, the lab effect, especially when confounded with the RNA sample effect, plays a bigger role than the platform effect on data agreement.
\end{abstract}

\section{Background}

Diversity of microarray data poses some unique and interesting questions on cross-experiment comparisons and the analysis tools needed for such comparisons. Since the invention of the microarray technology in 1995 [1], statistical methods and data mining techniques specific for microarray data have mushroomed [2], many of which have been packaged into commercial software such as GeneSpring and Spotfire. Such tools are useful for handling individual experiments, including quality control, significance testing, and clustering. However, researchers have questioned whether studies across different labs and technology platforms will have an acceptable level of agreement.

Possible incompatibility of results between similar microarray experiments is a major challenge that needs to be addressed, even though the data produced within a single experiment may be consistent and easy to analyze. Different labs produce microarray data in different ways using 
Table I: Summary of data collection

\begin{tabular}{ccccccccc}
\hline Data Set & Array & Genes & Sample & Replicates & Data type & Platform & Lab & Data Source \\
\hline KC & Cl I5K cDNA & $15 K$ & Clonetech & 4 & Raw intensity & cDNA & Keck & In house \\
KAV & Affymetrix 430A & $23 \mathrm{~K}$ & Clonetech & 2 & AV(Bioconductor) & Affymetrix & Keck & In house \\
KLW & Affymetrix 430A & $23 K$ & Clonetech & 2 & Li and Wong & Affymetrix & Keck & In house \\
KRMA & Affymetrix 430A & $23 K$ & Clonetech & 2 & RMA & Affymetrix & Keck & In house \\
CC & Riken I6K cDNA by Agilent & $16 K$ & Clonetech & 3 & Raw intensity & cDNA & Cal Tech & NCBI GEO \\
CO & Riken I6K Oligo by Agilent & $16 K$ & Clonetech & 3 & Raw intensity & Oligo & Cal Tech & NCBI GEO \\
GNF & Affymetrix U74Av2 & $12 K$ & In house & 2 & AV(MAS4.0) & Affymetrix & GNF & expression.gnf.org \\
\hline
\end{tabular}

different technology platforms, such as Affymetrix GeneChip, spotted cDNA array, and spotted oligo array. Affymetrix GeneChip uses one fluorescent dye while the spotted array uses two fluorescent dyes in the experiments. Direct comparison of raw data obtained from different technologies may not be meaningful. Instead, the final form of the data is often presented as relative expression levels, mostly ratios of intensities, after some statistical treatments including filtering, normalization and model-based estimation. Experiments using different technologies require different protocols for analyzing the raw data to derive the ratios. Scientists have published microarray data in a variety of formats including raw intensities and ratios of intensities. Does it make a difference which technology platform is chosen? Can we make use of the studies from different platforms and labs? To answer these questions and provide some guidance for platform comparisons, we report on a comparative study of three different platforms. The experiment is a simple two-tissue comparison between mouse liver and spleen. We used previously published data sets from two different sources as well as new data sets produced in house. There are several similar studies published in recent years [3-8]. A noticeable difference of this study from earlier ones is that we considered lab as a major factor in the comparison. In addition, we compared three major types of technology platforms, namely Affymetrix GeneChip, spotted cDNA array and spotted long oligo array. This study aims to provide a basis for further development of methodologies for comparing microarray data across different experiments and for the integration of microarray data from different labs.

\section{Results}

\section{Data collection}

As summarized in Table 1, a total of five data sets were either collected from a public source or generated in house. The samples for the experiments were normal mouse liver and spleen RNA, which were purchased from Clonetech (Catalog No. 64042-1 liver; Catalog No. 64044-1 spleen) except for the data set GNF generated by Su et al. [9] at the Genomics Institute of the Novatis
Research Foundation. Detailed sample descriptions for the GNF data can be found at http://expression.gnf.org. Two data sets were downloaded from the NCBI Gene Expression Omnibus http://www.ncbi.nih.gov/geo/, which were generated by Choi et al. at California Institute of Technology (Cal Tech) using Agilent oligo (GEO accession: GSE334) and CDNA arrays (GEO accession: GSE330), respectively. Two other data sets were generated at the Functional Genomics Unit at the W. M. Keck Center for Comparative and Functional Genomics at the University of Illinois using an in-house printed cDNA mouse array and Affymetrix mouse expression set $430 \mathrm{~A}$, and the data sets are available at http://titan.biotec.uiuc.edu/ cross_platform/. Another data set was downloaded from http://expression.gnf.org and it was generated using Affymetrix Murine Genome set U74Av2. The data set names (e.g., KC for the cDNA data set generated at the Keck Center at the University of Illinois) given in Table 1 will be used throughout the paper.

\section{Consistency of replicates}

One indication of data reliability is the consistency of replicates in a particular data set. We used kappa coefficients as well as the Pearson correlation coefficients and intraclass correlation coefficients on the replicates within each data set. Those measures set a benchmark against which the reliability of different platforms can be assessed; see Figure 1. The data set KC has four replicates from double spots of each gene on the array and from the dye swap. Therefore, we can do six pairwise comparisons of replicates. The data sets $\mathrm{CC}$ and $\mathrm{CO}$ have three replicates each; therefore, there are three pairwise comparisons. All Affymetrix data sets have two replicates and thus only one comparison. From Figure 1, we see that the replicates were quite consistent within each technology. The replicates in all the data sets showed pairwise Pearson correlation coefficients of 0.80 or higher, intraclass correlation coefficients of 0.77 or higher, and kappa coefficients of 0.43 or higher. The data from the Cal Tech (CC and $\mathrm{CO})$ showed the highest agreement among the replicates, and the data from GNF and KAV showed a low level of 
Pearson Correlation Coefficients of Replicates

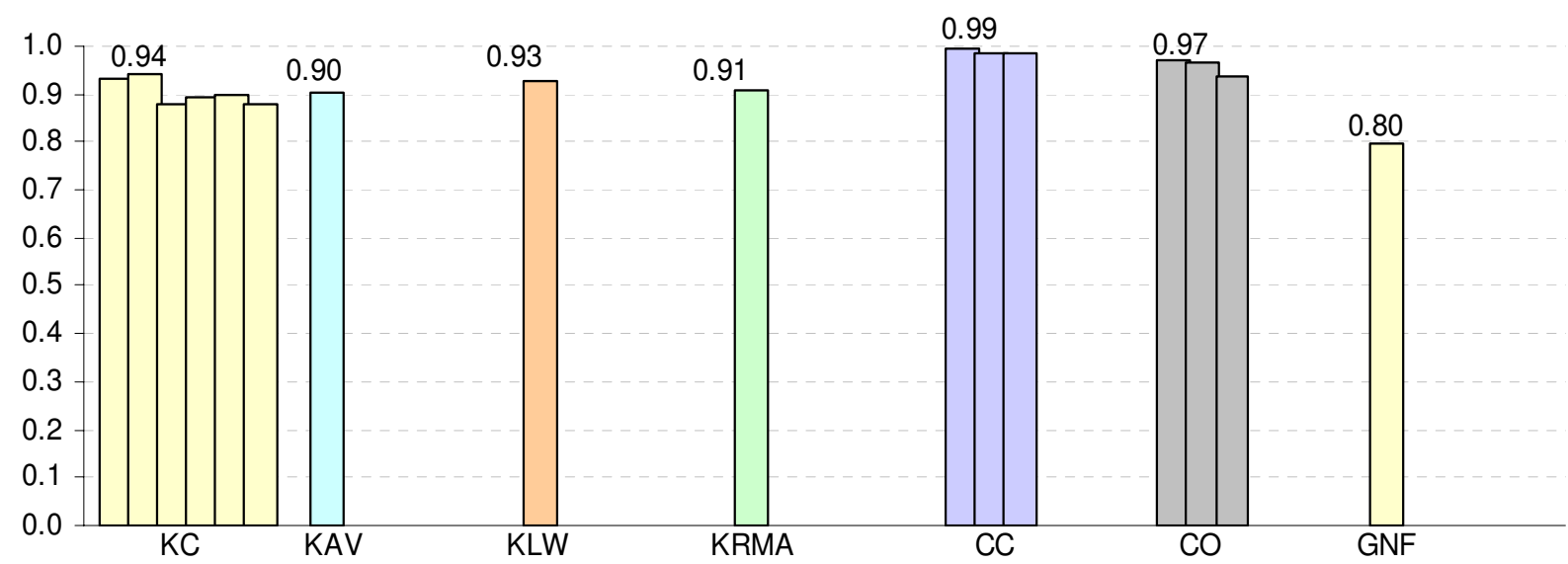

Two-fold Change Kappa Coefficients of Replicates

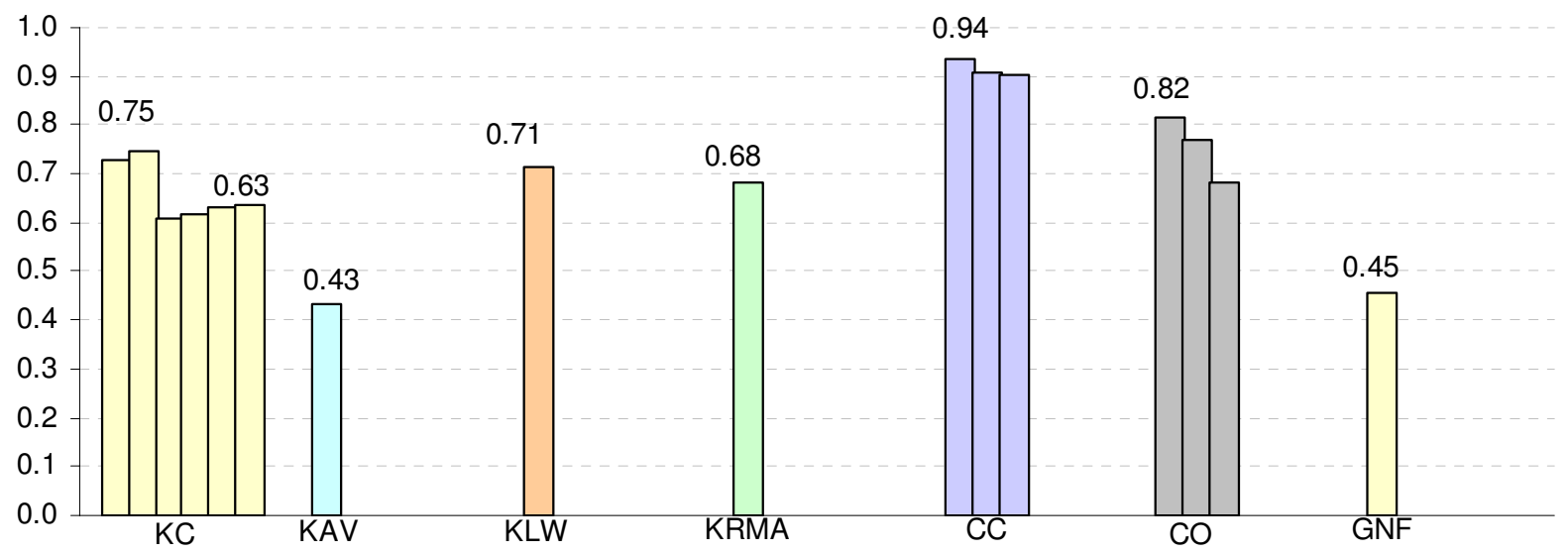

Intraclass Correlation Coefficients of Replicates

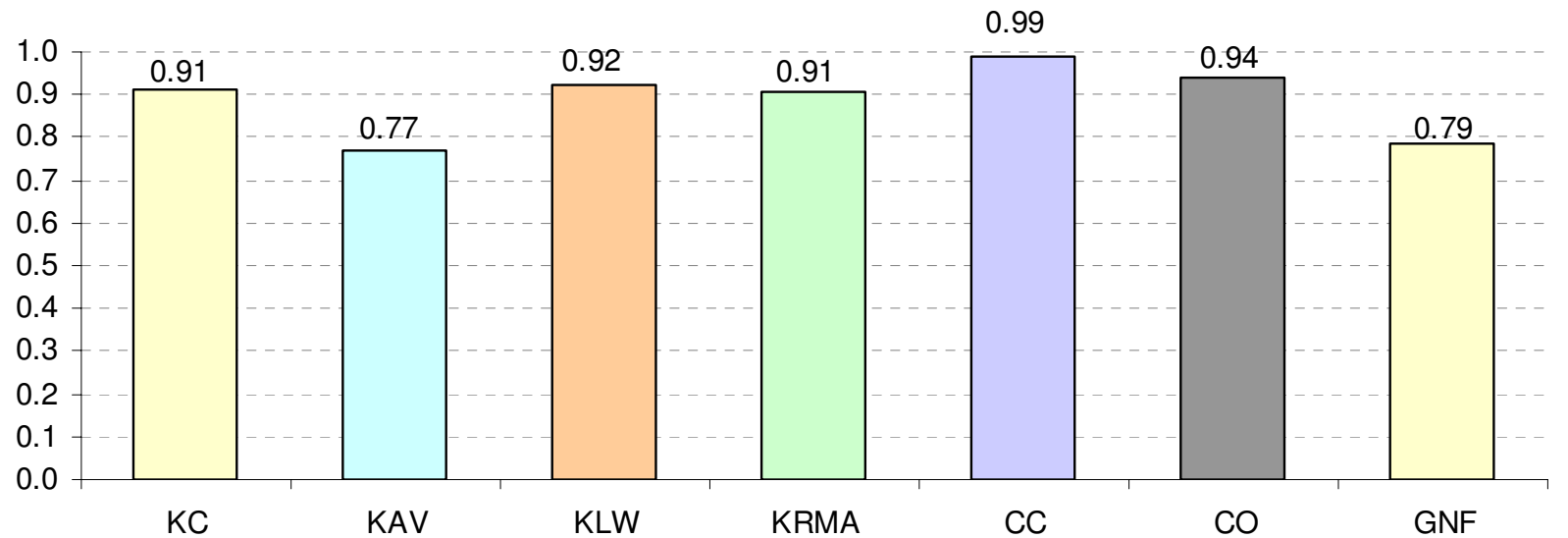

Figure I

Consistency of replicates. 
Table 2: Correlation coefficients for pairwise comparisons between data sets. Pearson correlation coefficients (PCC), kappa coefficients (Kappa), intraclass correlation coefficients (ICC) and intra-transcript correlation coefficients (ITC) for pairwise comparisons.

\begin{tabular}{|c|c|c|c|c|c|}
\hline Comparisons & $\begin{array}{l}\text { No. of Matched } \\
\text { Unigene IDs }\end{array}$ & PCC & Kappa & ICC & ITC \\
\hline GNF vs. KC & 1,838 & 0.590 & 0.327 & 0.693 & 0.748 \\
\hline GNF vs. CC & I,374 & 0.513 & 0.312 & 0.678 & 0.774 \\
\hline GNF vs. CO & $1,9 \mid 4$ & 0.633 & 0.365 & 0.707 & 0.729 \\
\hline GNF vs. KAV & 2,058 & 0.727 & 0.452 & 0.686 & 0.724 \\
\hline GNF vs. KLW & 3,295 & 0.640 & 0.374 & 0.681 & 0.690 \\
\hline GNF vs. KRMA & 3,452 & 0.686 & 0.400 & 0.706 & 0.705 \\
\hline KC vs. CC & 2,730 & 0.597 & 0.363 & 0.681 & 0.830 \\
\hline KC vs. CO & 3,043 & 0.641 & 0.423 & $0.7 \mid 4$ & 0.812 \\
\hline KC vs. KAV & 2,964 & 0.747 & 0.523 & 0.726 & 0.908 \\
\hline KC vs. KLW & 4,362 & 0.680 & 0.461 & 0.714 & 0.868 \\
\hline KC vs. KRMA & 4,516 & 0.725 & 0.493 & 0.736 & 0.893 \\
\hline CC vs. CO & 3,262 & 0.688 & 0.429 & 0.770 & 0.836 \\
\hline CC vs. KAV & 2,285 & 0.708 & $0.46 \mathrm{I}$ & 0.746 & 0.859 \\
\hline CC vs. KLW & 3,658 & 0.650 & 0.407 & 0.739 & 0.837 \\
\hline CC vs. KRMA & 3,843 & 0.707 & 0.472 & 0.772 & 0.862 \\
\hline CO vs. KAV & 3,001 & 0.806 & 0.555 & 0.781 & 0.865 \\
\hline CO vs. KLW & 4,725 & 0.759 & 0.503 & 0.782 & 0.847 \\
\hline CO vs. KRMA & 5,018 & 0.805 & 0.580 & 0.813 & 0.854 \\
\hline KAV vs. KLW & 7,181 & 0.923 & 0.666 & 0.832 & 0.917 \\
\hline KAV vs. KLW & 7,237 & 0.955 & 0.734 & 0.848 & 0.938 \\
\hline KLW vs. KRMA & 14,130 & 0.921 & 0.732 & 0.765 & 0.971 \\
\hline
\end{tabular}

agreement between replicates. These results suggest a lab effect in microarray data experiments.

For the KC data, we can see that two pairwise comparisons gave slightly higher agreement than the other four. It is, we believe, due to the double spots of each gene on the array. The two comparisons with higher agreement are the comparisons between the replicates within the slides.

\section{Pairwise comparisons among data sets}

Using the matched genes by common UniGene IDs, we compared different data sets in this study. Table 2 shows the Pearson correlation coefficients (PCC), intraclass correlation coefficients (ICC) and intra-transcript correlation coefficients (ITC) on log ratios, and the kappa coefficients (Kappa) for two-fold changes. The detailed descriptions of these measurements can be found in the "Methods" section. Figure 2 shows the histograms of the pairwise PCC, Kappa, ICC and ITC, respectively. With the exception of the GNF, most of the kappa coefficients are between 0.4 and 0.6 , as compared to 0.6 for the replicates within the KC data set.

From Figure 2 and Table 2, we see that the rankings of the pairwise comparisons are almost the same across four different measures of agreement. All the measures involving GNF are at the low end of the comparisons. The same is observed from the sensitivity check, which was done by leaving out one data set at a time and recording the changes of ICC as shown in Table 3. Excluding GNF resulted in the largest increase in ICC.

In a recent study, Jarvinen et al. [4] compared Affymetrix GeneChip, commercial cDNA array and a custom cDNA array using the same RNA samples from human cancer cell lines. They found that the data were more consistent between two commercial platforms and less consistent between custom arrays and commercial arrays. Their conclusion is consistent with our findings. In our study, $\mathrm{KC}$ is a custom cDNA array, whereas CC and CO are commercial cDNA and oligo arrays, respectively. If we do not consider comparisons involving GNF, we found that KC_CC (shorthand for KC versus CC) and KC_CO comparisons were ranked at the bottom. The samples used in $\mathrm{KC}, \mathrm{CC}$, and $\mathrm{CO}$ were identical, so biological variability is not an issue here. The variability among those data sets was mainly due to technical factors, such as platforms and labs conducting the experiments. Jarvinen et al. [4] analyzed the experiments conducted in one lab, and therefore their study was mostly concerned with the platform difference. Another study by Culhane et al. [3] used the co-inertia analysis to compare overall expression profiles across different platforms. Their analysis could be used on matched genes, as well as on all the data from different platforms. 
Pairwise Pearson Correlation Coefficients

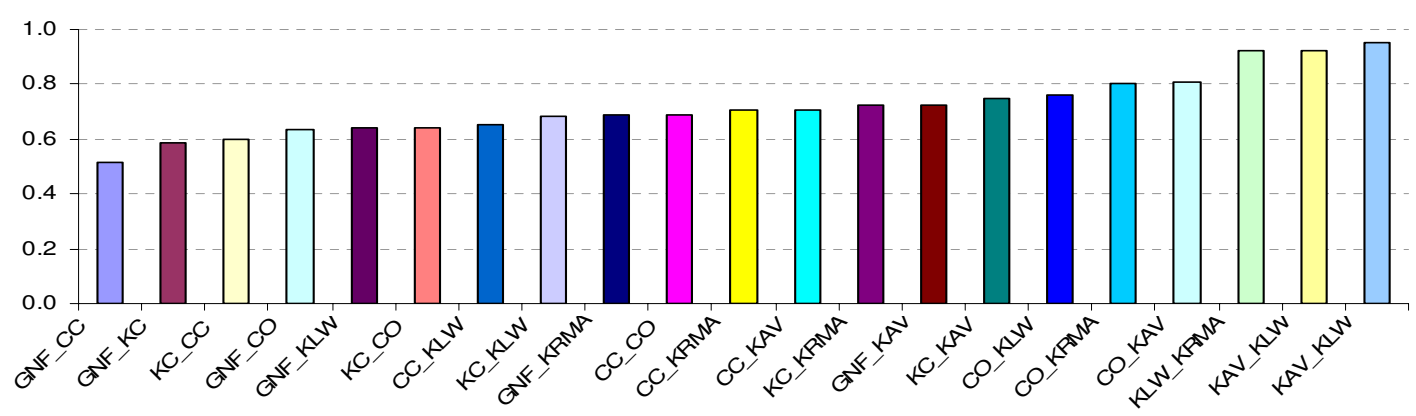

Pairwise Two-fold Change Kappa Coefficients

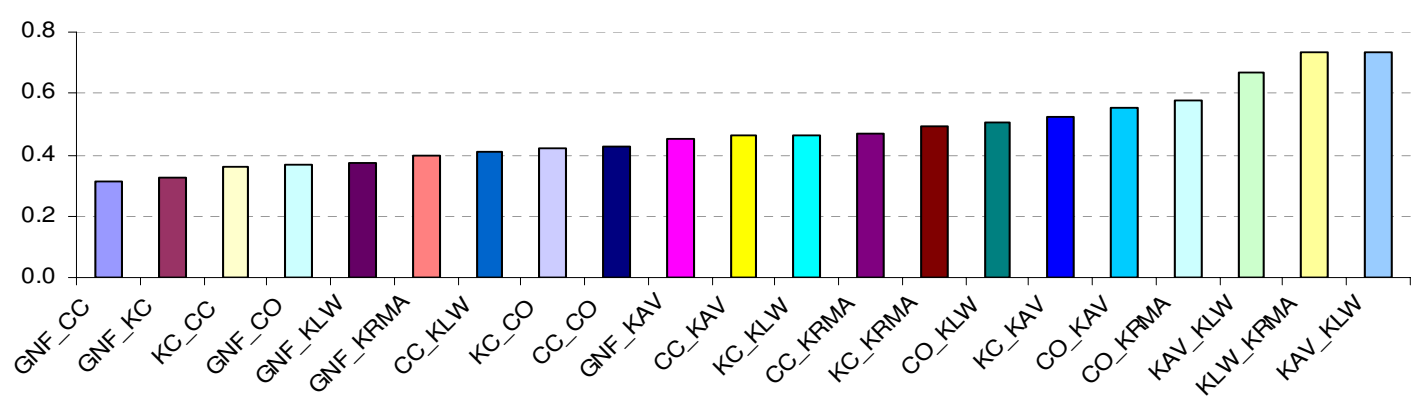

Pairwise Intraclass Correlation Coefficients

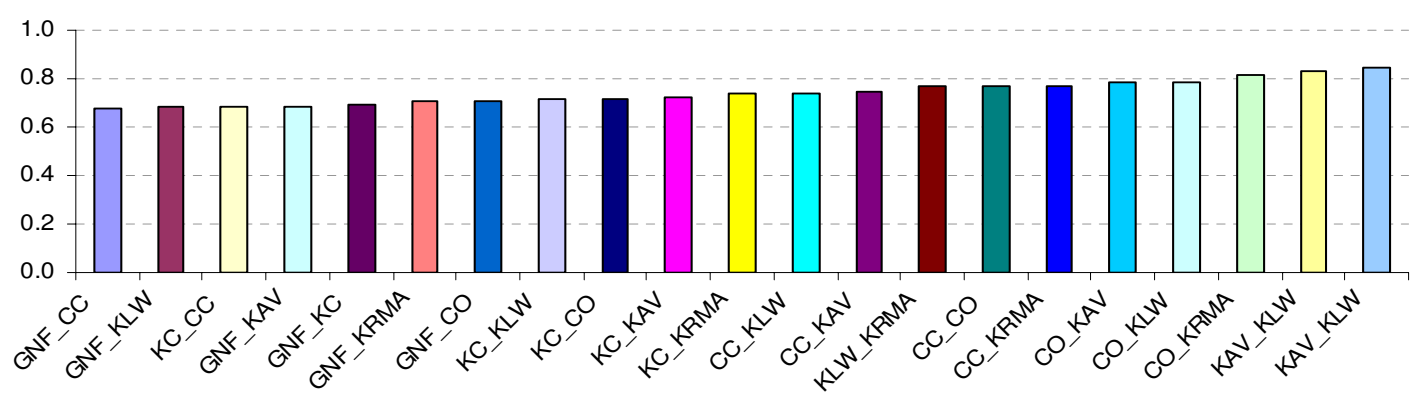

Pairwise Intra-transcript Correlation Coefficients

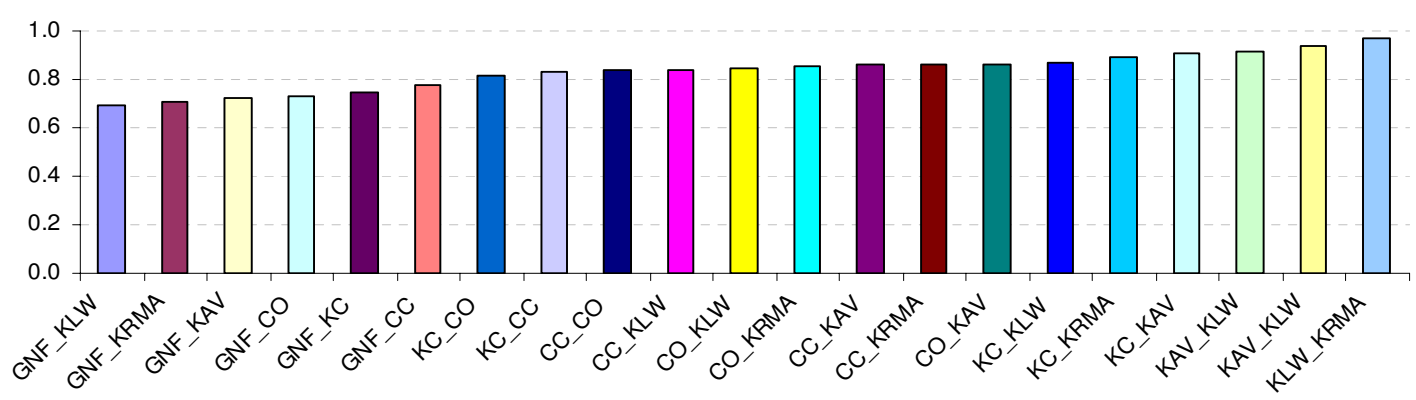

Figure 2

Correlation coefficients for pairwise comparisons between data sets. 
Table 3: Sensitivity check of the overall comparison among all the data sets

\begin{tabular}{ll}
\hline & ICC \\
\hline Before leaving out & 0.662 \\
Leave out GNF & 0.703 \\
leave out KLW & 0.650 \\
leave out KC & 0.663 \\
leave out CC & 0.684 \\
leave out CO & 0.670 \\
\hline
\end{tabular}

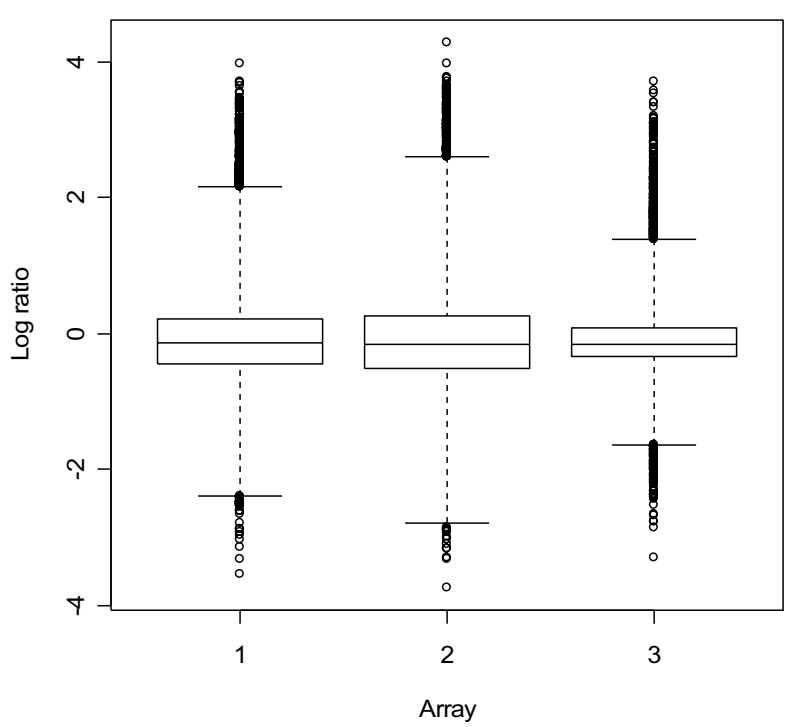

Figure 3

Boxplot of the full data set of CO, with 7,282 Unigene IDs.

While they considered the agreement for the overall expression profiles, we focused on the agreement in expressions at the gene level. Using the within-platform variability as a benchmark, we found that these technology platforms exhibited an acceptable level of agreement. For example, the overall comparison of all five data sets using KLW for the in-house Affymetrix gives ICC $=0.662$, as compared to the ICC around 0.8 for replicates within each data set. These results indicate that the agreement of different technologies is decent.

Since we only used a subset of genes in the study due to the gene matching problem, we asked whether the geneto-gene variation was impacted by the choice of the sub-

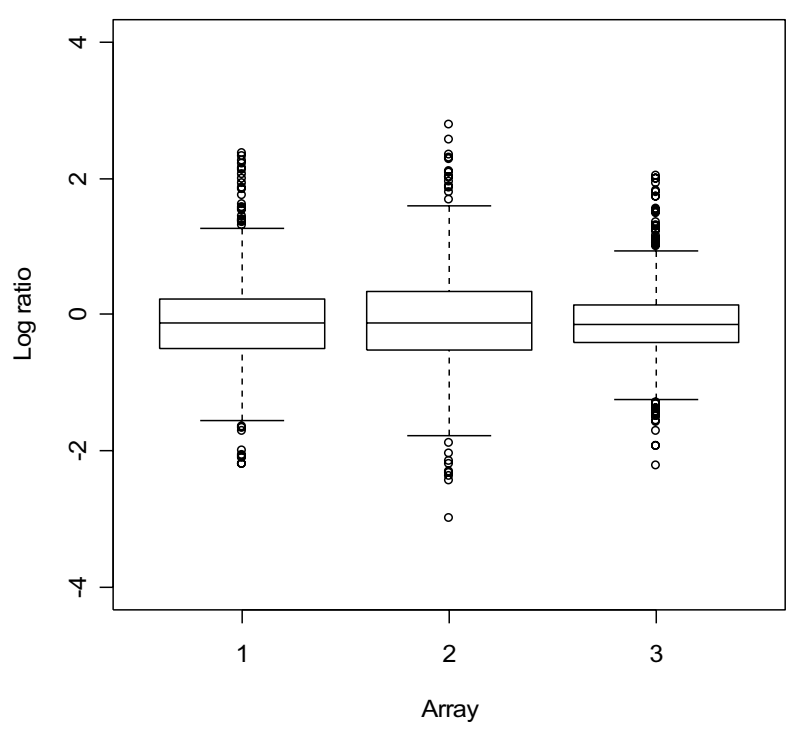

Figure 4

Boxplot of the subset of $\mathrm{CO}$, overlapped with the other 4 datasets, with 55 I Unigene IDs.

set. A comparison of the box plots for the full data set and for the subset used in the overall comparison showed that the variation in the subset of genes is similar to that of the full data set. For example, Figure 3 and Figure 4 give the box plots for the full data set and the subset of $\mathrm{CO}$, respectively. Therefore, we believe that a reasonable conclusion can be made based on the subset being used.

\section{Discussion}

UniGene has been widely used to match genes on different microarrays. Using UniGene and sequence similarity, Thompson, et al. [10] reported a number of gene markers that showed platform-independent expression profile. When we matched genes from different arrays using the mouse UniGene IDs, we found that there were multiple gene IDs in an array corresponding to one UniGene ID. Those genes were considered as "duplicate" genes, which made the cross matching of genes more complicated. A common approach is to average the expressions of those "duplicate" genes; however, we considered these "duplicate" genes as replicates in the technology and lab comparisons. One observation we should make is that the variability among these "duplicate" genes can be large. For example, in the CC arrays, there are 11,301 genes (upon filtering), corresponding to 8,318 UniGene IDs. Among the 8,318 UniGene IDs, 1,708 of them have "duplicate" genes. For the 12 Unigenes that have 10 or more 
"duplicate" genes, the ICC of the replicates decreased from 0.99 to 0.86 due to gene matching by Unigene IDs. Note that gene matching was performed only for the comparisons across data sets, and that we did not use the UniGene IDs in measuring the consistency within the same data set. This means that the agreement measures we obtained across different data sets were expected to be slightly lower than those from the replicates, even if the actual agreement was the same within or between data sets. In earlier studies, such as the one reported by Jarvinen et al., it has been shown that using different clones on different arrays is a major factor for the discrepancies between platforms. Using UniGene IDs to match the clones on different platforms can be problematic and result in biased comparisons. Sequence validation of the clones in different arrays may help resolve some of the problems and make the data from different platforms and labs more comparable.

From the present study, we showed that the GNF data set had the lowest agreement with the other data sets. This difference is compounded with the facts that the consistency of replicates within the GNF data set is the lowest and the sample used to generate the data is different from those used by other labs. We believe that the technology platform plays a relatively minor role in the disagreement, but the variation introduced by sample differences is one of the major factors. It has been shown that the expression level can vary significantly between genetically identical mice [11]. Variation among different individuals can be a significant factor for sample differences. Our analysis also indicates that data generated from different labs may have different quality even among the replicates, and thus quality control is important.

In this study, we also found that the lab effect can be greater than the platform effect. As shown in Figure 5, the comparisons between two different technology platforms in the same lab (KC_KRMA and CC_CO) showed better agreement than between two labs using the same technology (KC_CC). We also showed that the different summarization methods for Affymetrix exhibited good agreement.

Obviously, the present study has limitations. The results were generated from a very limited number of data sets. Using UniGene IDs for gene matching across data sets can also be questioned. Further research is clearly needed to address these limitations.

\section{Conclusion}

In this paper, we aim to address several issues in comparing microarray data across different platforms and different labs. We demonstrated that the consistency of replicates in each experiment varied from lab to lab. With

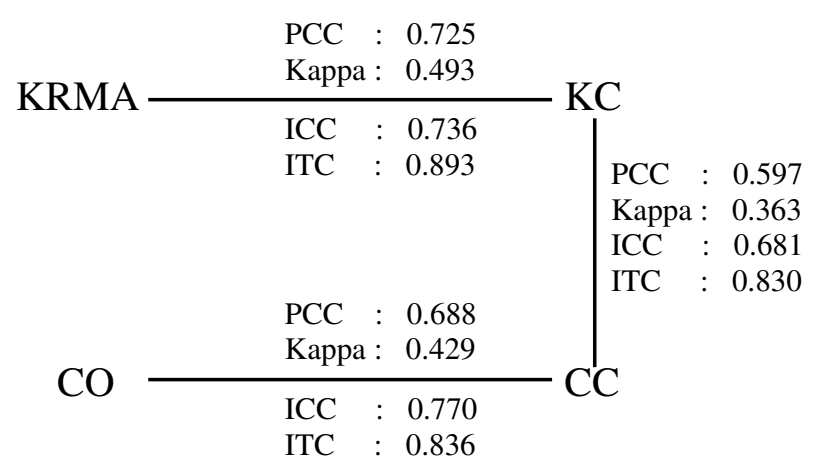

Figure 5

Comparisons between the same technology but different labs (KC_CC) and comparisons between different technologies in the same lab (KRMA_KC and CO_CC).

high consistency among replicates, different technologies seemed to show good agreement within and across labs using the same RNA samples. A closer look at the results indicated that the variability between two labs using the same technology was higher than that between two technologies within the same lab. The source of RNA samples can make a difference in microarray data, however in our present study we do not show conclusive results pertaining to possible sample or lab effects, because we did not have data collected from two different samples within one lab.

\section{Methods \\ Data processing}

For the spotted arrays (KC, $\mathrm{CC}$, and $\mathrm{CO}$ ), we used raw intensity data from both $\mathrm{Cy} 5$ and $\mathrm{Cy} 3$. We filtered out the non-expressive data points using median plus three times median absolute deviation (MAD, [12]) of the negative control genes as a criterion. We then performed global lowess normalization on each slide. For the KC data, we also performed paired-slide normalization following the method in Yang et al. [13] because of the dye swap in the experiment.

For the in-house Affymetrix data, we used three summarization methods to generate the probe set level signals. KAV and KRMA are based on the R package affy of Bioconductor using the average difference (AV) between Perfect Match (PM) and Mis-Match (MM) probe pairs, and the Robust Multi-Array Average (RMA) expression measure developed by Irizarry et al. [14], and KLW is the modelbased expression indexes developed by Li and Wong [15]. The GNF Affymetrix data were available to us only in the format of average differences. For both GNF and in-house 
Affymetrix probe set signals, we performed global lowess normalization for the three pairwise combinations of the liver and spleen slides, and used the averaged lowess adjustment for the normalization. The genes with probe set signals lower than 20 in either liver or spleen tissue were filtered out.

To stabilize the variances of data across the full range of gene expressions, we also performed the generalized log transformation for all the data sets following Durbin et al. [16].

\section{Gene matching across arrays}

There are five different data sets in this study. The origins of the genes vary in those datasets. In order to study interlab agreement, we have to first identify common genes represented in different arrays. Based on the annotation of each data set, we found that we could maximize the number of cross-matched genes using the mouse UniGene IDs (Build 107). Based on the common UniGene IDs, we found 551 common genes across all five different data sets. But in the pairwise comparisons, the number of common genes ranged from 1,374 to 5,018 (see Table 2). All comparisons between data sets were made from the matched genes.

\section{Statistical procedures for inter-platform and inter-lab comparisons}

In the analyses, the ratio is defined as normalized and transformed intensity from liver samples versus that from spleen samples.

\section{Agreement of two-fold changes using kappa coefficients}

An intuitive measurement of agreement is to count the percentage of genes falling in the same categories (twofold up-regulated, no change, and two-fold down-regulated). However, this percentage can be high even if the data obtained from different platforms are not so compatible. Usually the ratios for the great majority of genes do not show a two-fold change, and the percentage of agreement can be high just due to chance. To adjust for this excess agreement expected by chance, we prefer to use the kappa coefficient, which is a popular measure of interrater agreement in many other areas of science. The kappa coefficient was first proposed by Cohen [17] for analysing dichotomous responses, and was extended later to more than two categories of responses. We applied this measure to three categories (two-fold up-regulated, no change, and two-fold down-regulated), and computed the kappa coefficients between two data sets from 3 by 3 frequency tables. For a study of $q$ categories, the kappa coefficient is calculated by: kappa $=\frac{P_{a}-P_{e}}{1-P_{e}}$, where $P_{a}=\frac{1}{n} \sum_{k=1}^{q} n_{k k}$ is
Table 4: Frequency table for KAV and KC

\begin{tabular}{lllll}
\hline KAV & \multicolumn{3}{c}{ KC } \\
\hline Frequency & -2 & 0 & 2 & Total \\
-2 & 173 & 136 & 5 & 314 \\
0 & 157 & 1,972 & 146 & 2,275 \\
2 & 3 & 112 & 260 & 375 \\
Total & 333 & 220 & 411 & 2,964 \\
\hline
\end{tabular}

Kappa coefficient $=0.523$ the overall agreement probability, $P_{e}=\sum_{k=1}^{q} \frac{n_{+k}}{n} \cdot \frac{n_{k+}}{n}$ is the measure of the likelihood of agreement by chance, and $n_{i j}$ is the number of subjects in the $(i, j)$ cell, $n_{i+}$ is the sum of the $i$ th row, $n_{+j}$ is the sum of the $j$ th column, and $n$ is the total number of subjects.

For example, the kappa coefficient between KC and KAV is 0.523 . Table 4 gives the two-fold gene regulation frequency table between $\mathrm{KC}$ and KAV. Except for the 8 genes that showed two-fold up-regulation in one data set but two-fold down-regulation in another, $\mathrm{KC}$ and KAV agreed very well.

\section{Correlation coefficients of the ratios}

We used three measures of correlation to compare the ratios from different data sets: Pearson correlation coefficient, intraclass correlation coefficient (ICC) and intratranscript correlation coefficient (ITC). ICC measures the inter-rater reliability relative to the total variability of the ratios. Here, a rater could be a replicate or a technology platform. ICC is the variance of different ratios between UniGene IDs, $\sigma_{b}^{2}$, divided by the total variance $\sigma_{T}^{2}$. A high ICC (close to 1 ) means that the inter-rater ratios vary little relative to the overall variability in the data. In computing the ICC for the replicates, $\sigma_{T}^{2}$ equals $\sigma_{b}^{2}+\sigma_{e}^{2}$, where $\sigma_{e}^{2}$ is the variance within UniGene IDs. If we consider lab as a random effect in the overall comparison, the total variance $\sigma_{T}^{2}$ will equal $\sigma_{b}^{2}+\sigma_{c}^{2}+\sigma_{e}^{2}$, where $\sigma_{c}^{2}$ is the variance between labs. The ICC incorporates both the association between raters and the rater differences, while the Pearson correlation is insensitive to the latter.

We introduced the ITC for pairwise comparisons as described below. For each gene $i$, we defined $\rho_{i}$ to be the square root of the ratio of within dataset sum of squares (SSW) and the total sum of squares (TSS). A common SSW was used in comparing lab pairs to avoid the problem of having seemingly higher ITC's due to unusually 
large within-lab variability at some lab. We applied logit transformation to each $\rho_{i}$ to get $\gamma_{i}$, and then calculated the average $\gamma$. Converting $\gamma$ back to the correlation scale, we obtained ITC $=\frac{e^{r}}{1+e^{r}}$.

\section{Authors' contributions}

HW and LL collected data from public resources. MB and $\mathrm{CW}$ generated in-house data. HW and LL processed the collected data. HW and XH did statistical analyses. All authors read and approved the manuscript.

\section{Acknowledgements}

This study was supported in part by the NIH Grant No. 2 P30 AR4I94010. We would like to acknowledge Al Bari for his work on printing the mouse cDNA array at the Keck Center, and thank the referees for helpful comments that led to improvements of the present paper.

\section{References}

I. Schena M, Shalon D, Davis RW, Brown PO: Quantitative monitoring of gene expression patterns with complementary DNA microarray. Science 1995, 270:467-470.

2. Quackenbush J: Computational analysis of microarray data. Nat Rev Genet 200I, 2(6):418-427.

3. Culhane AC, Perriere G, Higgins DG: Cross-platform comparison and visualisation of gene expression data using co-inertia analysis. BMC Bioinformatics 2003, 4:59.

4. Jarvinen A, Hautaniemi S, Edgren H, Auvinen P, Saarela J, Kallioniemi $O$, Monni $O$ : Are data from different gene expression microarray platforms comparable? Genomics 2004, 83: I I64- I I 68.

5. Kuo WP, Jenssen T, Butte AJ, Ohno-Machado L, Kohane IS: Analysis of matched mRNA measurements from two different microarray technologies. Bioinformatics 2002, 18:405-4I2.

6. Yuen T, Wurmbach E, Pfeffer RL, Ebersole BJ, Sealfon SC: Accuracy and calibration of commercial oligonucleotide and custom cDNA arrays. Nucleic Acids Res 2002, 30:e48.

7. Kothapalli R, Yoder SJ, Mane S, Loughran TP: Microarray results: how accurate are they? BMC Bioinformatics 2002, 3:22.

8. Li J, Pankratz M, Johnson JA: Differential gene expression patterns revealed by oligonucleotide versus long cDNA arrays. Toxicol Sci 2002, 69:383-390.

9. Su Al, Cooke M, Ching KA, Hakak Y, Walker JR, Wiltshire T, Orth AP, Vega RG, Sapinoso LM, Moqrich A, Patapoutian A, Hampton GM, Schultz PG, Hogenesch JB: Large-scale analysis of the human and mouse transcriptomes. Proc Natl Acad Sci 2002, 99(7):4465-4470.

10. Thompson KL, Afshari CA, Amin RP, Bertram TA, Car B, Cunningham M, Kind C, Kramer JA, Lawton M, Mirsky M, Naciff JM, Oreffo V, Pine PS, Sistare FD: Identification of platform-independent gene expression markers of cisplatin nephrotoxicity. Environmental Health Perspectives 2004, I I 2:488-494.

II. Pritchard CC, Hsu L, Delrow J, Nelson PJ: Project normal: Defining normal variance in mouse gene expression. Proc Natl Acad Sci 200I, 98: | 3266-|327|.

12. Huber PJ: Robust Statistics. Cold Spring Harbor, John Wiley \& Sons; 1981.

13. Yang YH, Dudoit S, Luu P, Speed TP: Normalization for cDNA microarray Data. In Microarrays: Optical Technologies and Informatics SPIE BIOS San Jose, CA; 200I.

14. Irizarry RA, Hobbs B, Collin F, Beazer-Barclay YD, Antonellis KJ, Scherf U, Speed TP: Exploration, Normalization, and Summaries of High Density Oligonucleotide Array Probe Level Data. Biostatistics 2003, 4:249-264.

15. Li C, Wong WH: Model-based analysis of oligonucleotide arrays: Expression index computation and outlier detection. Proc Natl Acad Sci 200I, 98:3I-36.

16. Durbin BP, Hardin JS, Hawkins DM, Rocke DM: A Variance-Stabilizing Transformation for Gene-Expression Microarray Data. Bioinformatics 2002, I8:SI05-SIIO.
17. Cohen J: A coefficient of agreement for nominal scales. Educational and Psychological Measurement 1960, 20:37-46.
Publish with Biomed Central and every scientist can read your work free of charge

"BioMed Central will be the most significant development for disseminating the results of biomedical research in our lifetime. " Sir Paul Nurse, Cancer Research UK

Your research papers will be:

- available free of charge to the entire biomedical community

- peer reviewed and published immediately upon acceptance

- cited in PubMed and archived on PubMed Central

- yours - you keep the copyright
BioMedcentral 